\title{
Model of polarization conductivity in ionically conducting glasses
}

\author{
J. C. GIUNTINI, F. BUET, F. HENN, J. V. ZANCHETTA and J. VANDERSCHUEREN*
}

Université Montpellier II, Laboratoire de Physico-Chimie des Matériaux Solides, (URA D 407 CNRS), 34095 Montpellier Cedex 5, France

"Université de Liège, Chimie Macromoléculaire et Chimie Physique, SART TILMAN, 4000 Liège, Belgium

\begin{abstract}
We have used a hopping conduction model to calculate the frequencydependent polarization conductivity, $\sigma^{\prime}(\omega)$ in ionically conducting glasses, and we have assumed that a short-range ionic hopping mechanism occurs based on a fluctuation process. We have underlined the fundamental differences between the frequencydependent polarization conductivity due to "bound-charge" and the dc conductivity related to "free-charge" diffusion. The law A $\omega^{\mathrm{s}}$ is observed as for electronic chargecarriers in amorphous substances. The expression of parameter $s$ is modified by the introduction of a term connected with the fluctuation probability. A plot of the function $T / 1-s=f(T)$ shows a striking difference in behaviour (slopes) between ionic glasses $\left(\mathrm{Na}_{2} \mathrm{O}-3 \mathrm{SiO}_{2},\left(\mathrm{Ag}_{2} \mathrm{~S}\right)_{x}\left(\mathrm{GeS}_{2}\right)_{1-x}\right)$ and electronic amorphous solids.
\end{abstract}

1) Introduction. The use of ionic conducting glasses has been a subject of great interest for ten years $(1,2)$, and charge motion processes in these solids have been the matter of many investigations $(3,4)$. A well-known experimental technique has been frequently used to study these kind of solids : complex impedance spectroscopy (5). Originally it allowed information on the direct current conductivity measured on ionic conductors to be obtained (6).

Later on, this technical approach proved very interesting in the evaluation of the local order of amorphous substances. Qualitative and quantitative information is obtained thanks to the interpretation of the polarization conductivity, $\sigma^{\prime}(\omega)$, as a function of the frequency, $f(7)$ :

$$
\sigma^{\prime}(\omega)=A \omega^{5} ; \omega=2 \pi f
$$

A is a constant and $s$ a parameter experimentally determined which will be discussed later. Indeed, this study leads to the value of the experimentally accessible conductivity, $\sigma_{\text {meas.' }}$ which is the sum of two components, $\sigma_{d c}$ and $\sigma^{\prime}(\omega)$, such as :

$$
\sigma_{\text {meas. }}=\sigma_{d c}+\sigma^{\prime}(\omega)
$$

This equation simply comes from the well-known relation :

$$
\operatorname{div} \vec{E}=\frac{\rho}{\varepsilon_{0}}-\operatorname{div} \frac{\vec{P}}{\varepsilon_{0}}
$$


where $E$ is the electric field, $\rho$ the density of "free-charge", i.e. contributing to the dc conductivity, $\mathrm{P}$ the polarization, and $\varepsilon_{0}$ the dielectric constant.

2) The polarization conductivity. The polarization conductivity is not concerned by the "free-charge" movements because these charges tend to be opposed to the existence of the electric field in the substance (Faraday's law). The charges responsible for the polarization conductivity are necessarily "bound-charges". The corresponding charge carriers are therefore localized within sites where their movements are limited to small oscillations around their equilibrium position. Sometimes the carrier undergoes a short distance hop towards a neighbouring site. This situation describes a fluctuation phenomenon. Such a picture suggests the representation of the sample as a population of dipoles, each hop being similar to a dipole reorientation (7). In the absence of an electric field all the dipoles are oriented at random. The resulting momentum is zero. The field creates a reorientation of the dipoles : consequently a macroscopic polarization appears. If an alternating electric field is used, the dielectric dipole moment is out of phase with the electric field, and a polarization conductivity takes place. Thus the conductivity appears as the result of the movement of the ions around their equilibrium position.

3) The model. In this approach the polarization conductivity is therefore the ratio of the current density of the polarization process to the applied electric field. For a given frequency, $\sigma^{\prime}(\omega)$ is proportional to the hopping distance R,due to the "bound-charge'". This process depends on $(8,9,10)$ :

- the probability of finding an acceptor site from a given site i.e. $N p(R) d R$, where $N$ is the number of sites per unit volume, and $p(R) d R$ the spatial distribution characterizing the nature of the medium.

- the dipole polarizability : $\alpha(R)=e^{2} R^{2} / 12 k T$

- the resonance phenomenon which connects the frequency of the field to the relaxation time, $\tau$, characteristic of the hop. This leads to :

$$
\sigma^{\prime}(\omega)=\int N \alpha(R) p(R) \frac{\omega^{2} \tau}{1+\omega^{2} \tau^{2}} d R
$$

The integral can be evaluated if a relation between $R$ and $\tau$ is proposed $(11,12)$. Indeed, the first model comes from Debye's theory. The "pair-approximation" (PA) models are based on the following hypothesis : the sample is described as a collection of Debye independent relaxation processes (13). Especially in the case of the correlated barrier hopping (CBH) model, the relaxation time is : $\tau=\tau_{0} \exp (W / k T)$, with $\tau=1 / w(14), w$ being the probability, for a charge carrier, to hop over a potential barrier $W$. For an isotropic medium, $W=W_{M}-e^{2 / \varepsilon R}$ (15) $\left(W_{M}\right.$ : energy required to move an ion from its site to infinity).

Looked at from that point of view, the CBH model can be qualified as "perfect" in terms of the absence of interaction between the elementary components of the system. These theoretical considerations lead to (16): 


$$
\sigma^{\prime}(\omega)=A \omega^{s} \quad \text { with } s=1-\frac{6 k T}{W_{M}+k T \ln \left(\omega \tau_{0}\right)}
$$

( $\tau_{0}:$ phonon relaxation time)

Obviously, $T / 1-s=f(T)$ is a linear function. For electronic charge carriers, for which the PA model has been developed, the slope of the straight line is of the order of -3 .

In the case of ionic glasses the behaviour of the polarization conductivity is still, $\sigma^{\prime}(\omega)=A \omega^{\mathrm{s}}$ (17). This analogy leads to the assumption that the elementary microscopic process is of the same kind. However, analysis of the experimental results shows that parameter s does not behave as for electronic charge carriers.

An approach based on the $\mathrm{CBH}$ model, using the Anderson and Stuart potential barrier (18) has already been developed $(2,19)$.

A general explicative scheme consists of considering that the interactions between dipoles create a modification of the behaviour of $s$. For the same purpose we propose a description of the solid where an ion can only move if the thermal motion is sufficient. This means that we take into account the fluctuations of the medium as a possible explanation of the difference with the so-called "perfect" system. The fluctuations around the equilibirum state imply shortdistance hopping displacements. It is known that these kinds of fluctuations are correlated. This hypothesis is supported by measurements of thermally stimulated current, performed with blocking electrodes (Fig. 1). In this example, the important role of the thermal energy in the ionic transport phenomenon appears clearly.

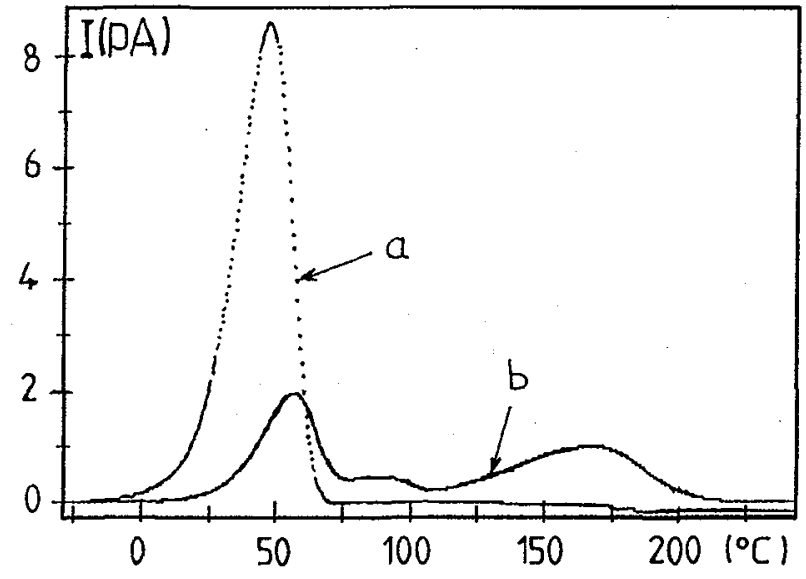

Fig 1 Spectra of thermally stimulated samples:
(a) $\mathrm{GeS}_{2}+\mathrm{Ag}_{2} \mathrm{~S}$
(b) pure $\mathrm{GeS}_{2}$

Correlations between the fluctuations are taken into account in the expression of the energies. So we propose an approach based on a two-component potential barrier as for the previous models $(2,19)$. From a phenomenological point of view, it is known that, when the fluctuations are considered, the probability involved is simply connected, through the entropy, to the minimum work i.e. the Gibbs function (20): $1 / \tau=w \sim \exp \left(-\Delta G_{\mathrm{p}} / k T\right)$.

As charges are still implied, the second term is related to the electrostatic energy, and the probability is modified. The total energy is : $W=W_{M}+\Delta G_{f}-e^{2 / \varepsilon R}$, with $\tau=\tau_{0} \exp (W / k T)$. 
Taking into account this new form of $W$, the calculation shows that : $\sigma^{\prime}(\omega)=A \omega^{5}$ and $s=1-6 k T /\left(W_{m}+\ln \left(\omega \tau_{0}\right)\right)$ with $W_{m}(T)=W_{M}+\Delta G_{f}$

Although $\Delta G_{f}$ has been introduced by means of macroscopic considerations, we do not prejudge its evaluation which can be microscopic (statistical thermodynamic) (19).

Table 1 Values of the slopes of the function $T / 1-\mathrm{s}=\mathrm{f}(\mathrm{T})$ for typical electronic and ionic amorphous conductors.

\begin{tabular}{|l|c|c|c||c|c|c|}
\hline sample & $\mathrm{AlPO}_{4}$ & $\mathrm{MOS}_{2+\mathrm{x}}$ & calc $^{*}$ & $\left(\mathrm{Ag}_{2} \mathrm{~S}_{0,5}\left(\mathrm{GeS}_{2}\right)_{0,5}\right.$ & $\left(\mathrm{Ag}_{2} \mathrm{~S}_{0,3}\left(\mathrm{GeS}_{2}\right)_{0,7}\right.$ & $\mathrm{Na}_{2} \mathrm{O}-3 \mathrm{SiO}_{2}$ \\
\hline slope & $\begin{array}{c}-3.04 \\
\text { to }\end{array}$ & $\begin{array}{c}-2.85 \\
\text { to }\end{array}$ & $\begin{array}{c}-2.37 \\
\text { to }\end{array}$ & -1.01 & -1.31 & +0.23 \\
\hline
\end{tabular}

*Theoretical evaluation eq(1): $W_{M}=1.3 \mathrm{eV} ; \tau_{0}=10^{-12}$ to $10^{-14} \mathrm{~s} ; \omega=210^{6} \mathrm{rad}^{-1}$

4) Conclusion. Table 1 reports, for amorphous electronic solids, the values of the slopes of the experimental results plotted in the form of $T / 1-s=f(T)$. The same procedure used for amorphous ionic glasses leads to very different values (Table 1). This fact justifies the approach adopted, by simply introducing an additional term in the expression of the potential barrier and therefore by modifying the equation of $s$. What seems interesting is that this description of the elementary process of polarization conductivity allows the extention of the concept of "bound-charge" to amorphous ionic glasses.

\section{References}

1. K.L. NGAl, Comments on Sol. Stat. Phys. 90 (1980) 141.

2. S.R. ELLIOTT and F. HENN, J. Non-Cryst. Solids $116(1990) 179$.

3. S.R. ELLIOTT, Solid State Ionics $\underline{27}$ (1988) 131.

4. K.L. NGAI, Solid State lonics $\underline{5}$ (1981) 27.

5. J.R. MC DONALD, Impedance Spectroscopy (J. WILEY and Sons, New York, 1987).

6. G. WILLIAMS and D.C. WATTS, Trans. Faraday Soc. $\underline{66}$ (1970) 80.

7. A.K. JONSCHER, Dielectric Relaxation in Solids (Chelsea Dielectric Press, London, 1983).

8. N.F. MOTT and E.A.DAVIS, Electronic Processes in Non-Cryst. Materials (Clarendon Press, Oxford, 1979).

9. S.R. ELLIOTT, Adv. Phys. 36 (1987) 135.

10. M. POLLAK, Phil. Mag. 23 (1971) 519.

11. J.C. GIUNTINI, J.V. ZANCHETTA and F. HENN, Solid State lonics 28-30 (1988) 142.

12. J.C. GIUNTINI, B. DEROIDE, P. BELOUGNE and J.V. ZANCHETTA, Solid State Comm. 62 (1987) 739.

13. M.P.J. van STAVEREN, H.B. BROM and L.J. de JONGH, Physics Reports 208, 1 (1991) 1.

14. M. POLLAK and T.H. GEBALLE, Phys. Rev. 122 (1961) 1742.

15. S.R. ELLIOTT , Phil. Mag. 36 (1977) 1291.

16. A.R. LONG, Adv. Phys. 31 (1982) 553.

17. D.P. ALMOND and A.R. WEST, Solid State lonics 9 (1983) 277.

18. O.L. ANDERSON and D.A. STUART, J. Am. Ceram. Soc. 37 (1954) 573.

19. F. BUET, J.C. GIUNTINI, F. HENN and J.V. ZANCHETTA, Phil. Mag. (in press).

20. L.D. LANDAU and E.M. LIFSHITZ, Physique Statistique (MIR Editions, 1967). 